\title{
KILOMETER-WAVE TYPE III BURST: HARMONIC EMISSION REVEALED BY DIRECTION AND TIME OF ARRIVAL
}

\author{
HECTOR ALVAREZ, FRED T. HADDOCK, and WILLIAM H. POTTER \\ Radio Astronomy Observatory University of Michigan, Ann Arbor, Mich. 48104, U.S.A.
}

(Received 26 June; in revised version 20 November, 1973)

\begin{abstract}
A type III solar burst was observed at seven frequencies between $3.5 \mathrm{MHz}$ and $80 \mathrm{kHz}$ by the Michigan experiment aboard the IMP- 6 satellite. From the data we can determine burst directionof-arrival as well as time-of-arrival. We predict these quantities using simple models whose parameters we vary to obtain a good fit to the observations. We find that between $3.5 \mathrm{MHz}$ and $230 \mathrm{kHz}$ the observed radiation was emitted at the fundamental of the local plasma frequency while below $230 \mathrm{kHz}$ it was emitted at the second harmonic. The exciter particles that produced the burst onset and burst peak have velocities of 0.27 and 0.12 , respectively, in units of the velocity of light.
\end{abstract}

\section{Introduction}

The university of Michigan experiment aboard the OGO-5 satellite detected solar type III bursts at eight frequency channels in the range from $3.5 \mathrm{MHz}$ to $50 \mathrm{kHz}$ (Alvarez and Haddock, 1973). A study of 64 strong events revealed that, in most cases, there was an unexpectedly long delay in the burst arrival times between two adjacent channels. A simple interpretation was possible by proposing the hypothesis that the observed radiation changed from the fundamental to the second harmonic of the local plasma frequency. It was found then that the radiation observed at hectometric and kilometric wavelengths was predominantly second harmonic(Haddock and Alvarez 1970, 1973). Some of the implications of this hypothesis are that the heliocentric distance of the burst sources is nearly doubled and that the bursts are not necessarily produced in dense streamers. This hypothesis has recently received theoretical (Zaitsev et al., 1972; Smith, 1973) as well as observational support (Fainberg et al., 1972).

The University of Michigan experiment aboard the IMP-6 satellite can give information on time-of-arrival as well as direction-of-arrival of the bursts. Here we present the analysis of a type III burst observed by IMP-6 and show that the directional and temporal results lend support to the second harmonic hypothesis. Since the issue is not yet considered as resolved (Stone and Fainberg, 1973), the main purpose of this paper is to present more convincing evidence.

\section{The Experiment}

The Michigan radio astronomy experiment on the IMP-6 spacecraft is a stepping radiometer, operating on the eight frequencies of $3505,900,600,350,230,130,80$ and $50 \mathrm{kHz}$. Each channel is sampled every $5.12 \mathrm{~s}$. The bandwidth is $10 \mathrm{kHz}$; the detector time constant is $0.12 \mathrm{~s}$. The antenna is a dipole approximately $55 \mathrm{~m}$ from tip to tip. The spacecraft rotates with a period close to $12 \mathrm{~s}$ about an axis which is maintained 
perpendicular to the plane of the ecliptic within a few degrees. The two nulls of the dipole antenna pattern on the plane of rotation pass approximately through the Sun each revolution, imposing an amplitude modulation on the observed signals from which directional information may be derived. We will assume that the spin axis is perpendicular to the ecliptic.

\section{Data}

The time profile of a burst at a given frequency is divided into subintervals of specified length. The computer program assumes a sinusoidal modulation of known period and performs an independent least-squares fit to the observed data in each sub-interval to determine the value of source direction and depth of modulation which best fits. The program then uses these parameters to compute an antenna gain correction factor for each data point, and plots the 'demodulated' data.

The burst discussed here occurred on 20 January 1972 . It drifted from $3.5 \mathrm{MHz}$ down to $80 \mathrm{kHz}$ and in all but the lowest frequency it shows amplitude modulation. At $80 \mathrm{kHz}$ the burst was weak. This was one of the first events studied using the direction-of-arrival information; it was selected because of its simple structure (apparently a single burst) and because of the good signal-to-noise ratio. A weak burst started at 0214.7 UT in the $3.5 \mathrm{MHz}$ channel and it was followed by the main burst (about 100 times stronger) that started at 0219.0 UT. The small burst disappeared below $900 \mathrm{kHz}$.

There was only one optical flare whose onset occurred within 50 min of the $3.5 \mathrm{MHz}$ burst onset. It was a $-F$ flare located at S16, E06. Its onset, peak and end occurred at 0215,0217 and 0221 UT, respectively (Solar-Geophysical Data, N.O.A.A.).

\section{Analysis}

\subsection{Assumptions}

In the analysis we make the following assumptions:

(1) For the electron density distribution we used the three models obtained from the OGO-5 analysis (Alvarez and Haddock, 1973). However, to simplify the discussion, those models will be expressed here by the approximate formular:

$$
N=N_{E}\left(\frac{214}{r-1}\right)^{2.38} \text { for } \quad r \gtrsim 3.4 R_{\odot} \text {, }
$$

where $N_{E}\left(\mathrm{~cm}^{-3}\right)$ is the electron density at $1 \mathrm{AU}$ and $r$ is heliocentric distance in solar radii $\left(R_{\odot}\right)$. The errors in time-of-arrival and direction-of-arrival that would be introduced by this simplified formula are a fraction of $1 \%$ for the observing frequencies of our radiometer. The exponent was obtained from observations of type III burst frequency-drift-rate made over an eleven-year solar cycle.

(2) We assume that the exciter stream trajectory is an Archimedes spiral contained in 
the ecliptic plane. The equation of this curve is:

$$
\theta=\theta_{F}-\varepsilon\left(\frac{r}{215}\right)
$$

where $r$ is in solar radii. $\theta_{F}$ (deg) is approximately the heliographic longitude of the foot of the spiral on the Sun's surface and gives the orientation of the spiral with respect to the Sun-Earth line. The parameter $\varepsilon$, assumed constant, is the angular rotation of the spiral in $1 \mathrm{AU}\left(215 R_{\odot}\right)$ and it is inversely proportional to the solar wind velocity.

(3) In analyzing times-of-arrival we assume that each exciter particle travels along a spiral with constant velocity, expressed as the fraction $\beta$ of the velocity of light in vacuum. This assumption will be discussed later.

(4) We assume the local plasma hypothesis and allow for emission at both fundamental and second harmonic of the local plasma frequency. Following the definition used in a previous paper (Haddock and Alvarez, 1973) we will call the fundamental mode that radiation emitted at the fundamental of the plasma frequency in a coronal level where the electron density is, say, $N$ and we will call half-frequency harmonic mode (or simply, harmonic mode) that radiation emitted at the second harmonic of the plasma frequency in a further out level where the electron density is $0.25 \mathrm{~N}$. Both modes are observed then at the same frequency.

(5) We assume that the radio waves propagate from the source to the radiometer as in a vacuum. We define $\lambda$ as the angle, in the ecliptic plane, that the source makes with the Sun as seen from the Earth. All angles used in this work will be measured from the Sun-Earth line as reference and positively in a counterclockwise direction as seen from the north.

\subsection{FitTING PROCEDURE}

We have four adjustable parameters $\left(N_{E}, \theta_{F}, \varepsilon\right.$, and $\left.\beta\right)$ at our disposal, plus the decision to be made independently for each frequency as to whether the emission we see was generated at the fundamental or at second harmonic of the plasma frequency. In this analysis we restrict ourselves to only a few values for each parameter. We use three different electron density models: Model I $\left(\mathrm{N}_{E}=4.0 \mathrm{~cm}^{-3}\right)$, Model II $\left(\mathrm{N}_{E}=7.7 \mathrm{~cm}^{-3}\right)$, and Model III $\left(N_{E}=31.8 \mathrm{~cm}^{-3}\right)$. We use two values of $\varepsilon: 90^{\circ}$ and $60^{\circ}$ that correspond to solar wind velocities of 260 and $390 \mathrm{~km} \mathrm{~s}^{-1}$, respectively. We change $\theta_{F}$ in increments of $1^{\circ}$ and we vary $\beta$ in steps of 0.01 .

For each set of model parameters, and for each of our instrumental frequencies, we compute predicted direction-of-arrival and time-of-arrival for radiation generated as fundamental and half-frequency harmonic.

The general procedure is to find first which values of $N_{E}, \theta_{F}$ and $\varepsilon$ fit the observed direction-of-arrival data. This is independent of the value of $\beta$. Then, for several of the better fit cases we found values of $\beta$ that fit the observed time-of-arrival data as well. The time of burst onset and time of burst peak were fit separately. 


\section{Discussion of the Results}

The results of the best direction-of-arrival fit and best time-of-arrival fit for all frequencies were obtained for one set of parameters: $N_{E}=7.7 \mathrm{~cm}^{-3}, \varepsilon=60^{\circ}, \theta_{F}=-7^{\circ}$ $\pm 1^{\circ}, \beta=0.27 \pm 0.01$ for the burst onset, and $\beta=0.12 \pm 0.01$ for the burst peak. Figure 1 shows the direction-of-arrival fit. Note that here the observations fit the fundamental at and above $230 \mathrm{kHz}$ but they fit the half-frequently harmonic below that frequency. This general change from fundamental to harmonic emission at low frequencies was observed in the OGO-5 data (Haddock and Alvarez, 1973). In this particular event we cannot determine if the harmonic component was also present at higher frequencies, although in the OGO-5 events both were often present at the same frequency.

Figures 2 and 3 show the best time-of-arrival fit for burst onset and burst peak, respectively. Because the burst was weak at $80 \mathrm{kHz}$ only the onset time could be measured with reasonable accuracy. We notice that the mode sequence in which the observed times-of-arrival fit the predictions at the fundamental or harmonic emission is the same as in the direction-of-arrival fit. The consistency of this sequence in the three fits is strong evidence in support of the second-harmonic hypothesis. The trajec-

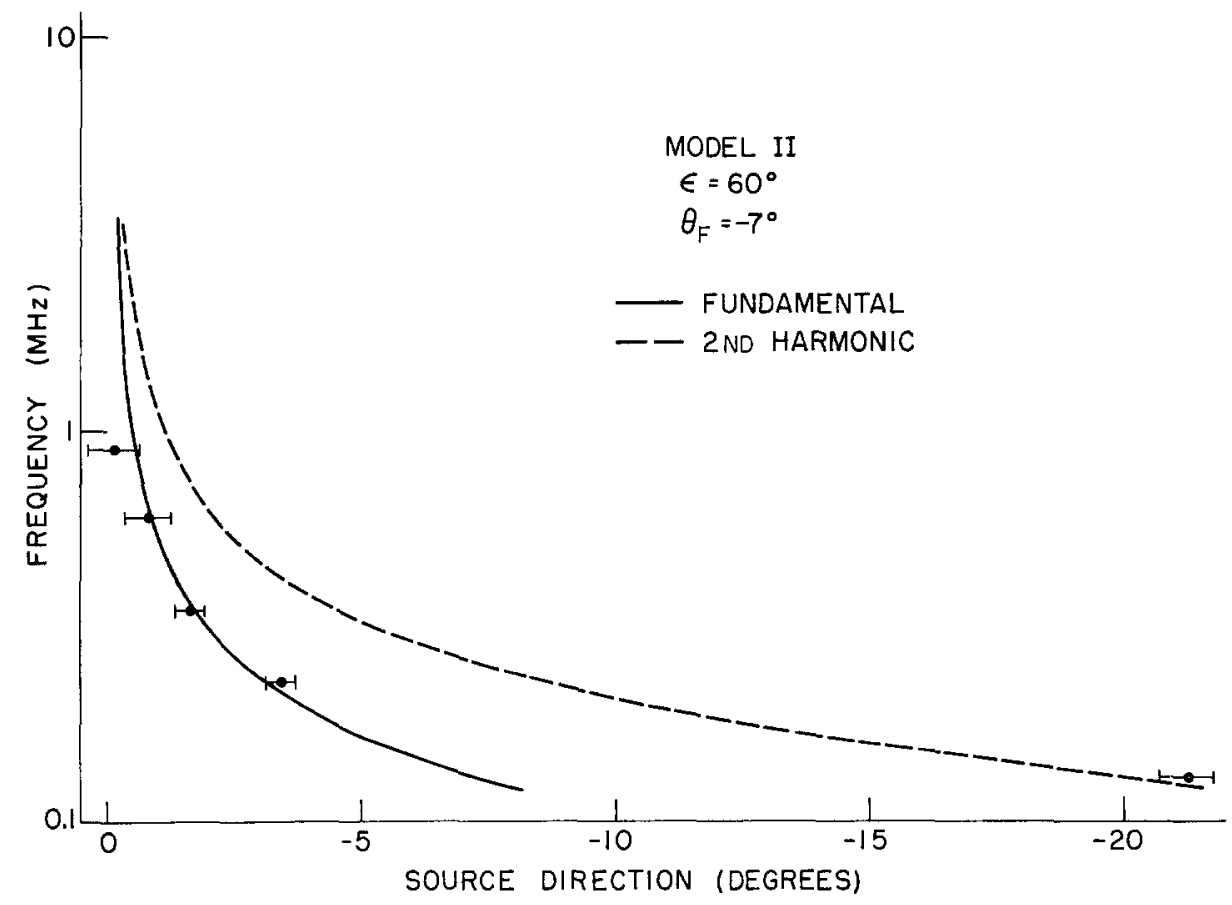

Fig. 1. Comparison of predicted and observed direction of arrival. The parameters used give the best fit; they are: electron density Model II $\left(N_{E}=7.7\right), \varepsilon=60^{\circ}$ and $\theta_{F}=-7^{\circ}$. It can be seen that above $130 \mathrm{kHz}$ the observation fit the fundamental while at $130 \mathrm{kHz}$ the observation fits the harmonic. 


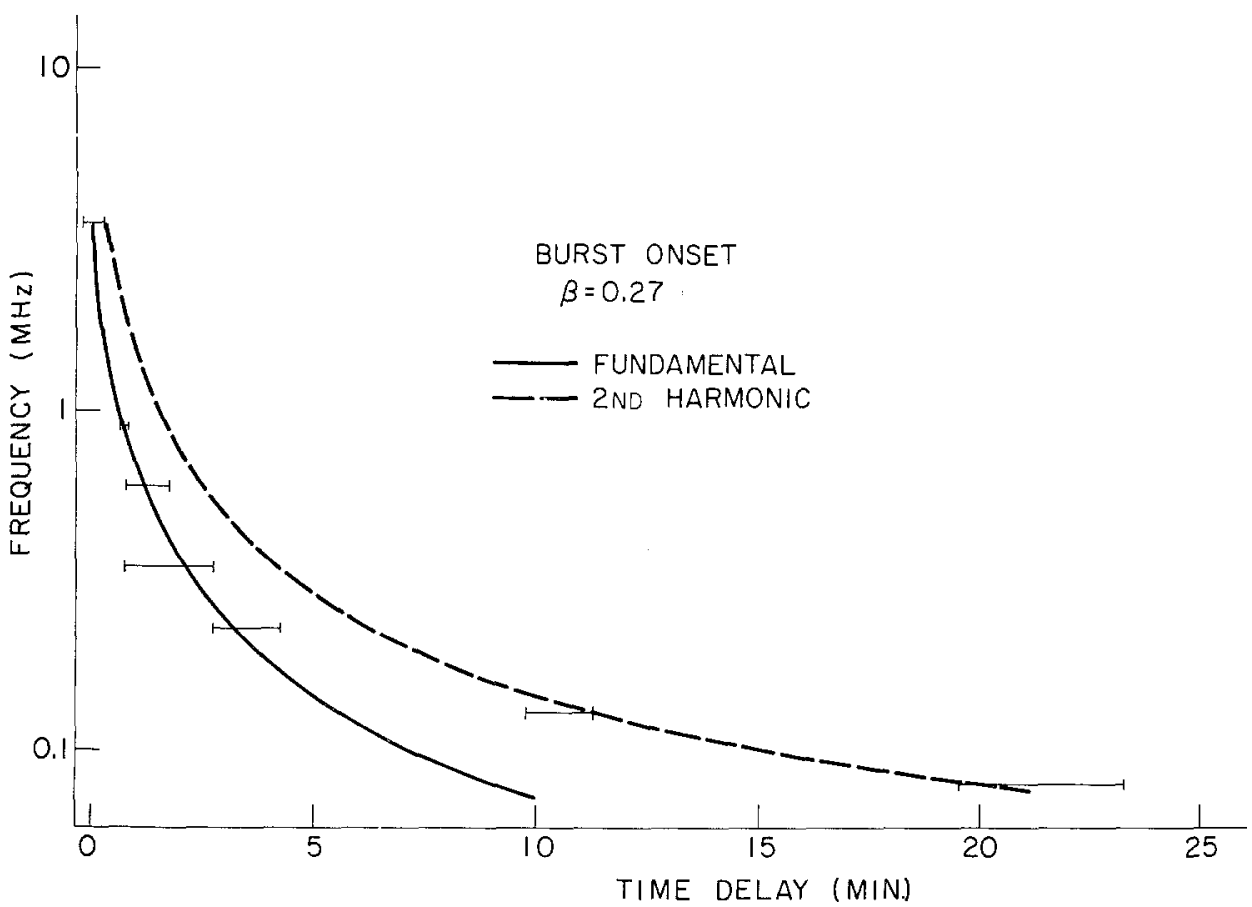

Fig. 2. Comparison of predicted and observed arrival times for the burst onset. The parameters used are the same for Figure 1. It can be seen that at 130 and $80 \mathrm{kHz}$ the observations fit the harmonic.

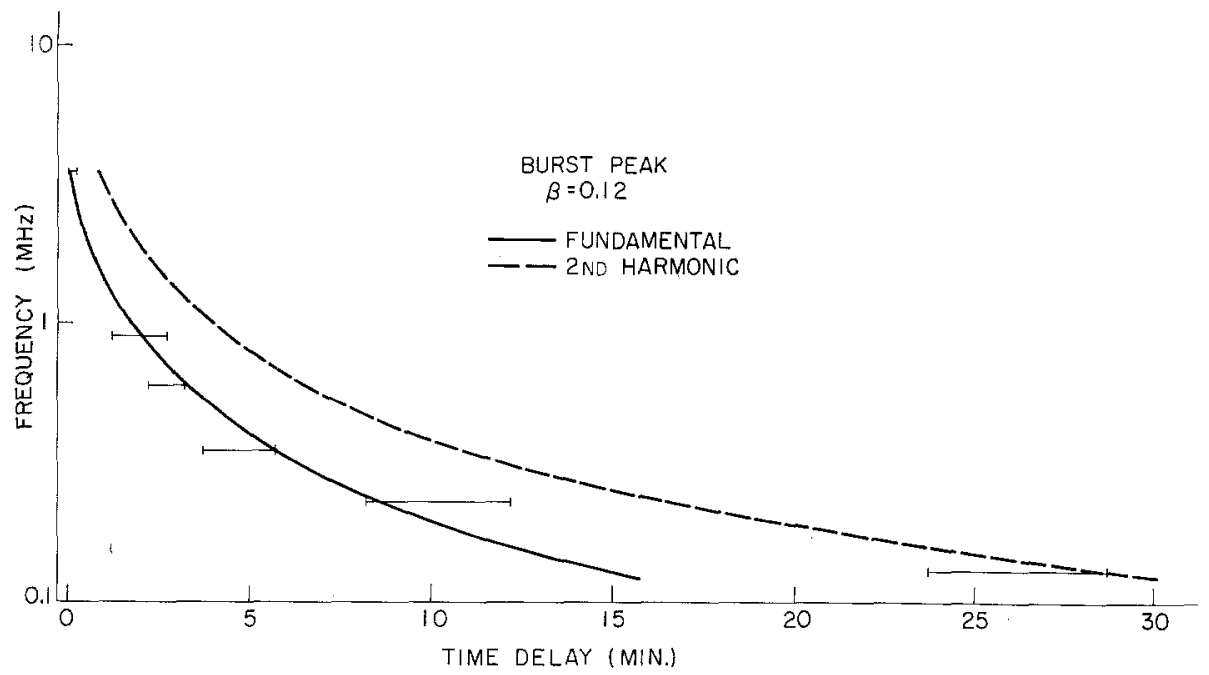

Fig. 3. Same as Figure 1 but for the arrival times of the burst peak. The consistency of the frequency sequence in which the observations fit the fundamental or harmonic predictions is the same in Figures 1, 2 and 3. This is interpreted as evidence that the radiation observed below $230 \mathrm{kHz}$ was second harmonic emission. The peak at $80 \mathrm{kHz}$ could not be measured. 
tory resulting from the best-fit parameters is illustrated in Figure 4 where the indicated frequencies are plasma frequencies. It is clear that a change from fundamental (230 $\mathrm{kHz}$ plasma level) to harmonic ( $65 \mathrm{kHz}$ plasma level) results in a 'jump' in the timeof-arrival as well as in the direction-of-arrival. The $50 \mathrm{kHz}$ burst (from the $25 \mathrm{kHz}$ plasma level) was not observed but in this model it should have occurred at $\lambda=-67^{\circ}$ and at a distance of $270 R_{\odot}$ from the Earth. It is not surprising that it is not observed since propagation effects would make it difficult for the radiation to reach the Earth.

The choice of the fitting parameters is supported by related observations. During the week following the burst, Vela 3 and 5 spacecraft made a few measurements of the solar wind density near the Earth. Values ranged between 2.3 and $5.6 \mathrm{~cm}^{-3}$. The solar wind velocity measured from the same spacecraft ranged between 384 and $547 \mathrm{~km} \mathrm{~s}^{-1}$ with the average near $470 \mathrm{~km} \mathrm{~s}$. The values of $7.7 \mathrm{~cm}^{-3}$ and $391 \mathrm{~km} \mathrm{~s}^{-1}$ given by the

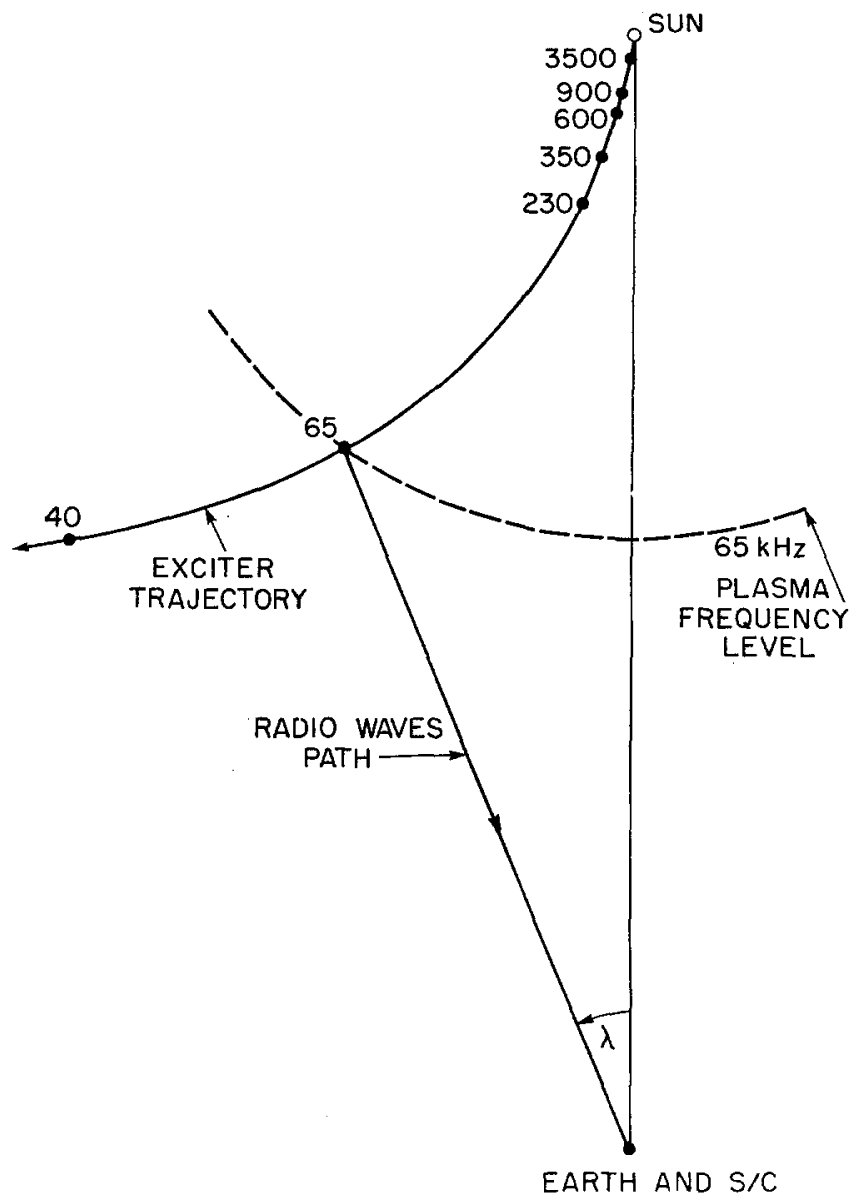

Fig. 4. Exciters stream trajectory and source positions resulting from the best fit. The parameters used are the same as in Figure 1. Frequencies shown are plasma frequencies. Our results indicate that the observed radiation of the bursts coming from the plasma levels of

65 and $40 \mathrm{kHz}$ was second harmonic. 
best fit model are reasonably close. The obtained $\theta_{F}=-7^{\circ}$ is in excellent agreement with the heliographic longitude $-6^{\circ}$ (E06) of the time associated flare previously described.

In order to further test the assumption that the $130 \mathrm{kHz}$ and $80 \mathrm{kHz}$ radiation is harmonic, we attempted to fit the $130 \mathrm{kHz}$ burst to fundamental emission. The observed direction-of-arrival for $130 \mathrm{kHz}$ indicates that the $130 \mathrm{kHz}$ plasma level is at a minimum heliocentric distance of $70 R_{\odot}$. Only Model III is dense enough to fulfill this condition. The resulting fit is poor and it is obtained for Model III $\left(N_{E}=31.8 \mathrm{~cm}^{-3}\right)$, $\varepsilon=90^{\circ}, \theta_{F}=10^{\circ}, \beta=0.30$ for the onset, and $\beta=0.14$ for the peak. We conclude that, within the framework of our analysis, the observed burst direction and time-of-arrival are inconsistent with the assumption that the 130 and $80 \mathrm{kHz}$ emissions are at the fundamental of the plasma frequency.

In assigning a velocity to the particles that produced the burst peak at the different frequencies, we have implicitely assumed that the burst rise time is determined by velocity dispersion in the exciter stream and not by plasma wave growth. This may be the case since at $130 \mathrm{kHz}$, for example, it takes the burst more than $12.5 \mathrm{~min}$ to rise to the maximum. Defining by $\bar{\beta}$ the mean stream velocity and by $\Delta \beta$ its dispersion, Smith (1970) infers a ratio $\Delta \beta / \bar{\beta}=0.50$ from Hughes and Harkness's observations (1963). Using our data we find a ratio of 1.3. From this result we can get a rough estimate of the linear extent of the exciter stream if we assume that the exciter particles started simultaneously from the Sun. By the time that the 0.12-particles reached the $65-\mathrm{kHz}$ plasma level (26 min of transit time) the leading edge of the stream consisting of 0.27 -particles is $100 R_{\odot}$ further out along the trajectory. This distance, already large, would represent a lower limit to the stream length.

The constancy of the exiters velocity for type III bursts is being debated. Fainberg et al. (1972) analyzed a kilometer wave type III burst using an electron density model and a trajectory determined by the closest distances to the Sun permitted by the observed directions of burst arrival. The authors interpreted their results as indicating that the exciter velocities derived for both burst onsets and burst peaks decreased by about a factor of two in almost 1 AU. However we take this result cautiously since their procedure restricts the exciter trajectory to lying on the circle, in the ecliptic plane, having the Sun-Earth line as a diameter, and since no argument is given for choosing this Earth-oriented trajectory.

We wish to emphasize that these analyses of space observations of type III bursts are obtained from a simple spinning dipole antenna and a multiple frequency receiver. The physical conditions of exciter trajectory, velocity, deceleration, propagation of the burst to the $S / C$ with scattering and refraction playing some unknown role are too complex to unravel with this simple experiment. We have therefore made what we believe to be the simplest and most reasonable assumptions. These assumptions do ignore the effects of irregular exciter trajectories and of radio wave scattering and refraction. As long as we obtain a good fit with a simple model and no conflicting evidence arises, we feel justified in assuming no deceleration for the exciter stream. 


\section{Conclusions}

Using simple assumptions we have analyzed the observation of a type III solar burst that drifted from $3.5 \mathrm{MHz}$ down to $80 \mathrm{kHz}$. The quality of the data is good and the measurements are reliable. We fitted the observed time-of-arrival and direction-ofarrival of the bursts at the different frequencies to those predicted by several models of electron density distribution, of exciter velocity and trajectory shape and orientation. We searched for the set of four parameters that would give a fit, and found that a complete fit of all data is possible only within narrow ranges of these four parameters.

We have shown that the burst could have been originated by a group of particles of which the ones producing the burst onset have a velocity of 0.27 and those producing the burst peak have a velocity of 0.12 . We have also shown that the particles could have traveled along magnetic field lines in an Archimedes spiral with $\varepsilon=60^{\circ}$ contained in the ecliptic plane, and starting about $7^{\circ}$ east of the central meridian of the Sun. The electron density distribution along the trajectory was close to that of Model II with $N_{E}=7.7 \mathrm{~cm}^{-3}$. Between $3.5 \mathrm{MHz}$ and $230 \mathrm{kHz}$ the emission was at the fundamental of the local plasma frequency while at 130 and $80 \mathrm{kHz}$ it was at the second harmonic from a level at a heliocentric distance of 97 and $146 R_{\odot}$ respectively. The $50 \mathrm{kHz}$ channel recorded no burst but, according to our model, this is to be expected.

We have shown that our observations at $130 \mathrm{kHz}$ and $80 \mathrm{kHz}$ cannot be reconciled with the type of models which we have used if the emission takes place at the fundamental of the plasma frequency.

The results support the hypothesis that in some type III bursts the observed radiation changes from fundamental to second harmonic in the hectometric or kilometric wavelength range.

\section{Acknowledgements}

We thank Dr M. F. Aller for helpful suggestions. This work was done under NASA contract NAS S-11174.

\section{Bibliography}

Alvarez, H. and Haddock, F. T.: 1973, Solar Phys. 29, 197.

Fainberg, J., Evans, L. G., and Stone, R. G.: 1972, Science 178, 743.

Haddock, F. T. and Alvarez, H.: 1970, Bull. Am. Astron. Soc. 3, 6.

Haddock, F. T. and Alvarez, H.: 1973, Solar Phys. 29, 183.

Hughes, M. P. and Harkness, R. L.: 1963, Astrophys. J. 138, 239.

Smith, D. F.: 1970, Adv. Astron. Astrophys. 7, 147.

Smith, D. F.: 1973, in R. Ramathy and R. G. Stone (eds.), 'High Energy Phenomena on the Sun', GSFC Symp. Proc., X-693-73-193; p. 558.

Stone, R. G. and Fainberg, J.: 1973, in R. Ramathy and R. G Stone (eds.), 'High Energy Phenomena on the Sun', GSFC Symp. Proc., X-693-73-193; p. 519.

Zaitsev, V. V., Mityakov, N. A., and Rapoport, V. O.: 1972, Solar Phys. 24, 444. 